\title{
Rendimientos del mercado accionario y depreciaciones cambiarias en México: 1988-2007
}

\author{
Domingo Rodríguez Benavides* y Arturo Morales Castro ${ }^{* *}$
}

\section{RESUMEN}

Conocer la forma en que los mercados financieros están relacionados es de suma importancia en la actualidad, en especial debido a que estas interrelaciones son decisivas en la transmisión o propagación de las crisis financieras. El propósito de este trabajo es investigar si las depreciaciones cambiarias inciden tanto en la media como en la varianza de los rendimientos accionarios, o en ambas. Para tal fin, empleamos la metodología propuesta por Siourounis (2004), la cual consiste en estimar un modelo GARCH con una variable exógena. Se encuentra evidencia empírica de que entre menor sea la magnitud de la depreciación cambiaria ésta sólo incide en la media de los rendimientos y no en su volatilidad; por el contrario, entre mayor sea la depreciación del tipo de cambio ésta impactará en la volatilidad del mercado accionario y no en los rendimientos promedio.

\section{Número de clasificación: JEL:}

Palabras clave: rendimientos accionarios, depreciaciones cambiarias, modelos GARCH.

\begin{abstract}
Knowing the form in which the financial markets are related is of great importance nowadays, specially due to the fact that these relationships are crucial in the transmission or propagation of the financial crises. The purpose of this paper is to investigate if monetary depreciation affects in the mean or the variance of stock market returns or both, using the methodology proposed by Siourounis (2004), which is the estimation of a GARCH model with a exogenous variable. Empiric evidence was found that lesser the magnitude of monetary depreciation, this impacts only on the mean returns not in its volatility, by contrary, greater the exchange rate depreciation, this impacts on volatility of the stock market and not in its mean returns.
\end{abstract}

Number of classification: JEL:

Keywords: stock returns, exchange rate depreciation, GARCH models.

\footnotetext{
*Facultad de Economía, UNAM <domr@economia.unam.mx>

***Facultad de Contaduría y Administración, UNAM <amcastro@correo.fca.unam.mx>
} 


\section{INTRODUCCIÓN}

En la esfera teórica existen varios vínculos entre el mercado cambiario y el mercado accionario. En el ámbito macroeconómico los inversionistas pueden considerar al mercado cambiario como una buena opción para invertir cuando el mercado accionario esté a la baja.

En el ámbito microeconómico puede darse un cambio en la composición de los portafolios de inversión y/o de la deuda de las empresas ante una devaluación, lo cual modifica el valor de sus activos. De lo anterior se desprende una relación de causalidad entre ambos mercados, en la cual en lo macro el cambiario sigue al bursátil y en lo micro el bursátil sigue al cambiario (Guzmán Plata et al., 2007). Sin embargo, en la bibliografía sobre la relación entre los mercados accionario y cambiario no se encuentran de manera explícita los mecanismos de transmisión entre ellos, ni entre sus principales indicadores: índice de precios y cotizaciones (IPC) y tipo de cambio.

En este contexto, y dada la volatilidad reciente experimentada en el ámbito financiero internacional, surge la necesidad de contar con una mayor cantidad de estudios que den cuenta de la interdependencia, de la forma en la que se encuentran interrelacionados y de los mecanismos de transmisión que existen entre los mercados accionario y cambiario, ya que generalmente en alguno de estos mercados es donde inicialmente se manifiestan las crisis económicas, propagándose de manera inmediata al resto de la economía.

En este trabajo intentamos investigar si las depreciaciones cambiarias inciden en diferente magnitud en los rendimientos promedio del mercado accionario y/o en su volatilidad, a partir de un modelo GARCH $(1,1)$ mediante la incorporación de una variable dummy, la cual da cuenta de la ocurrencia del evento. Básicamente, especulamos que entre mayor es la magnitud de la depreciación cambiaria ésta tiende a impactar en mayor medida en la volatilidad del mercado accionario, por lo que consideramos que la utilización de esta metodología puede revelar alguna posible relación sistemática entre ambos mercados.

Dado lo anterior, planteamos las siguientes preguntas: $i$ ) iel mercado accionario reacciona de forma inmediata a las depreciaciones del tipo de cambio?, si fuera el caso, ii) ¿reaccionan de la misma forma los rendimientos y su volatilidad ante las depreciaciones cambiarias de distinta magnitud, o bien lo hacen de forma asimétrica? Para responder estas interrogantes empleamos información histórica diaria de ambas variables para el periodo que comprende del 4 de enero de 1988 al 18 de julio de 2007. 
Se buscó que el periodo de estudio fuera el más amplio posible, debido a que cuando se analizan datos de alta frecuencia se recomienda que éstos abarquen el mayor lapso para inferir de manera más confiable las posibles interrelaciones que puedan existir entre ambas variables. Esta metodología se empleó también en la estimación de la incidencia de los eventos sociopolíticos en la rentabilidad y volatilidad del mercado accionario en México (Hernández et al., 2007).

\section{EL ENTORNO DE LOS MERCADOS ACCIONARIO Y CAMbiaRio EN MéXICO}

Una de las principales características del periodo de estudio es que en éste se registró un cambio en el régimen de tipo de cambio, que evolucionó de un tipo de cambio fijo en 1988 a un crawling peg con modesto deslizamiento y, finalmente, de enero de 1991 a diciembre de 1994, a la adopción de una banda que se ampliaba gradualmente.

En este esquema el tipo de cambio se ajustaría a las condiciones del mercado dentro de las fronteras de esta banda, lo cual permitía absorber fuertes presiones al alza y a la baja del mercado de divisas, pero a pesar de esto el régimen cambiario se desplomó ante el ataque especulativo desencadenado por los acontecimientos políticos de 1994 (Gil-Díaz y Carstens, 1996). Como consecuencia de la debacle cambiaria de fines de 1994, las autoridades monetarias pusieron a flotar el tipo de cambio para que éste se rigiera libremente por las fuerzas del mercado.

La relación entre estos dos mercados ha sido por demás evidente en el periodo de estudio. A pesar de lo anterior, no hay mucha investigación que aborde la relación entre el tipo de cambio y el índice de precios y cotizaciones y las posibles relaciones de causalidad entre estas variables diferentes.

Dentro de los estudios sobre este tema en México se encuentran los trabajos de Ibarrarán y Troncoso (1998) y Guzmán Plata et al. (2007), mientras que acerca de algunos países asiáticos se encuentra el de Granger, Huang y Yang (2000) y el de Qayyum y Kemal (2006) en el caso de Pakistán. Ibarrarán y Troncoso (1998) concluyen que el mercado accionario determina el mercado cambiario a partir de la estimación de un modelo VAR, mientras que Guzmán Plata et al. (2007) encuentran periodos breves de cointegración entre ambas variables para distintos subperiodos en la historia reciente de nuestro país, en los cuales la dirección de la causalidad cambia constantemente. 
GRÁFICA 1. Índice de precios y cotizaciones y tipo de cambio (4 de enero de 1988 a 18 de julio de 2007)

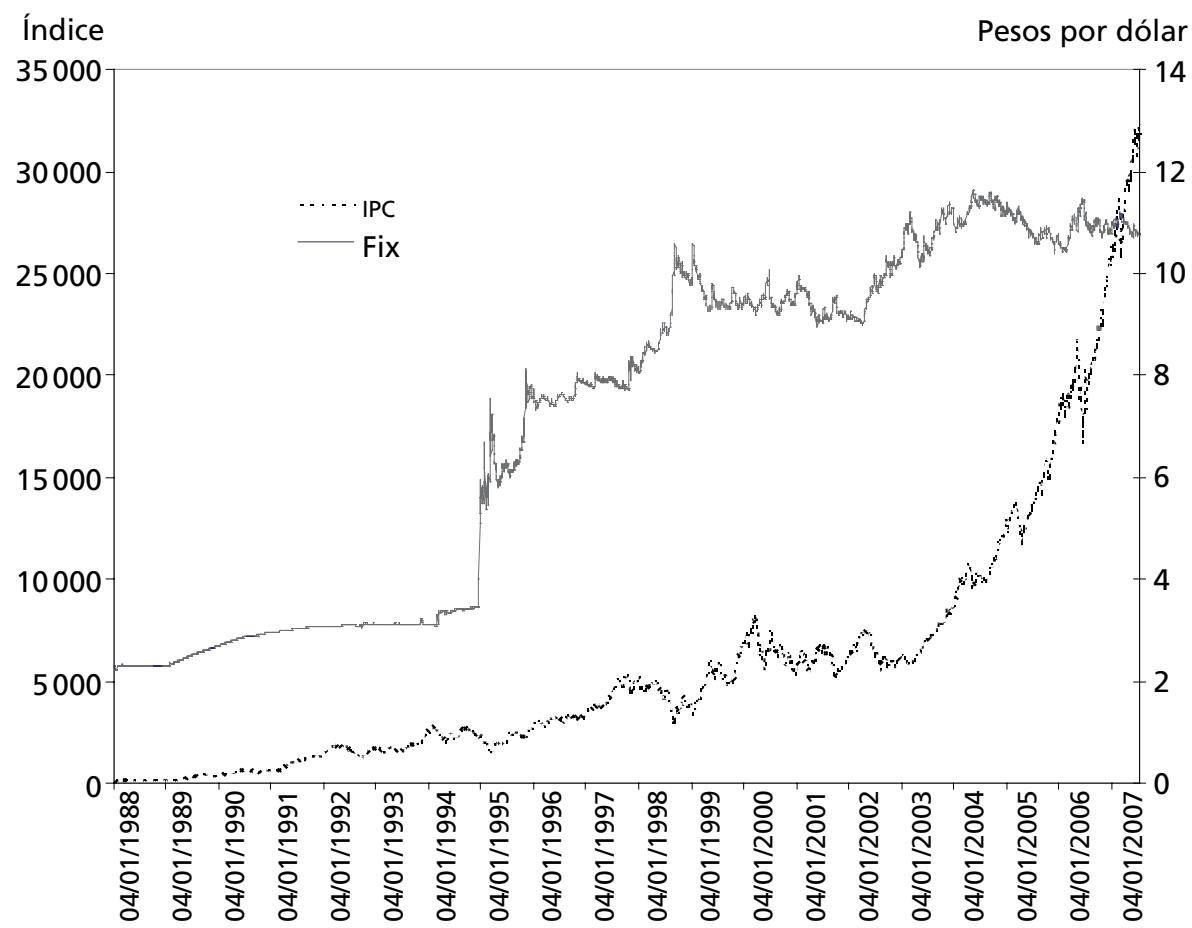

\section{Datos y hallazgos PRELiminares}

La gráfica 1 muestra la evolución del índice de precios y cotizaciones de la BMV y del tipo de cambio nominal en el periodo de estudio. Los rendimientos accionarios se calcularon como una transformación monotónica que no cambia el segundo momento de la serie:

$$
R_{t}=\log \left(P_{t} / P_{t-1}\right)
$$

Los días correspondientes a los días festivos fueron removidos de la serie de los rendimientos para evitar la incorporación de rendimientos espurios nulos. Con el fin de identificar si tanto la serie en niveles del IPC, como la de sus rendimientos a modelar poseen raíces unitarias se aplicaron las pruebas de DickeyFuller y Phillips-Perron. Acorde con la prueba Dickey-Fuller aumentada, la hipótesis de una raíz unitaria se prueba a partir del siguiente modelo:

$$
\Delta y_{t}=\alpha_{0}+\gamma y_{t-1}+\sum_{i=2}^{p} \beta_{i} \Delta y_{t+i+1}+\varepsilon_{t}
$$


con el fin de verificar si la hipótesis nula de $\gamma=0$. Si $\gamma=0$, se dice entonces que la serie en cuestión tiene una raíz unitaria. La prueba Phillips-Perron (PP) implica la estimación del modelo:

$$
y_{t}=\alpha_{0}+\alpha_{1} y_{t-1}+\alpha_{2}(t-T / 2)+u_{t}
$$

donde $T$ es el número de observaciones, el término de perturbación es tal que $E\left(u_{t}\right)=0$, y prueba la hipótesis nula de $\alpha_{1}=1 .{ }^{3} \mathrm{Si} \alpha=1$, entonces una raíz unitaria está presente en la serie. El cuadro 1 reporta las pruebas para las series $\log P_{t}$ y logaritmo de la primera diferencia $R_{t}$. Los resultados de la prueba ADF no indican la presencia de una raíz unitaria en el logaritmo de $P_{t}$ en ambos niveles de significancia, mientras que la prueba PP rechaza la presencia de una raíz unitaria en el nivel de $1 \%$, mas no a $5 \%$ de significancia en dicha serie. Tanto la prueba ADF como la PP para el $\log P_{t}$ se realizaron incorporando una tendencia e intercepto, el cual en ambos casos resultó significativo, mientras que las mismas pruebas para los rendimientos sólo incorporaron un intercepto.

CUADRo 1. Estadísticos preliminares para las series $\log P_{\mathrm{t}}$ y $R_{\mathrm{t}}$.

\begin{tabular}{|l|c|c|c|c|}
\hline Estadístico & \multicolumn{2}{|c|}{$\log P_{t}$} & \multicolumn{2}{|c|}{$R_{t}$} \\
\hline Observaciones & \multicolumn{2}{|c|}{4897} & \multicolumn{2}{|c|}{4896} \\
\hline Media muestral & \multicolumn{2}{|c|}{8.06442241395} & \multicolumn{2}{c|}{0.0011736339026} \\
\hline Varianza & \multicolumn{2}{|c|}{1.61519478686} & \multicolumn{2}{|c|}{0.0002724462643} \\
\hline Desviación estándar & \multicolumn{2}{|c|}{1.27090313827} & \multicolumn{2}{|c|}{0.0165059463321} \\
\hline Asimetría & \multicolumn{2}{|c|}{-0.61791161108} & \multicolumn{2}{|c|}{0.0174759358607} \\
\hline Curtosis & \multicolumn{2}{|c|}{2.89648053725} & 9.61321436675 \\
\hline Pruebas de raíces unitarias & Estadístico & Rezagos & Estadístico & Rezagos \\
\hline Dickey-Fuller (ADF) & $-4.0189 * *$ & 2 & $-47.46 * *$ & 1 \\
\hline Phillips-Perron (PP) & $-3.541 *$ & 1 & $-58.59 *$ & 0 \\
\hline
\end{tabular}

${ }^{*} \mathrm{y} * *$ denotan significancia a 5 y a $1 \%$.

\footnotetext{
${ }^{3}$ En esta prueba no se requiere que el término de perturbación no esté serialmente correlacionado o que sea homogéneo (Phillips y Perrron, 1988).
} 
GRÁFICA 2. Variaciones diarias del tipo de cambio

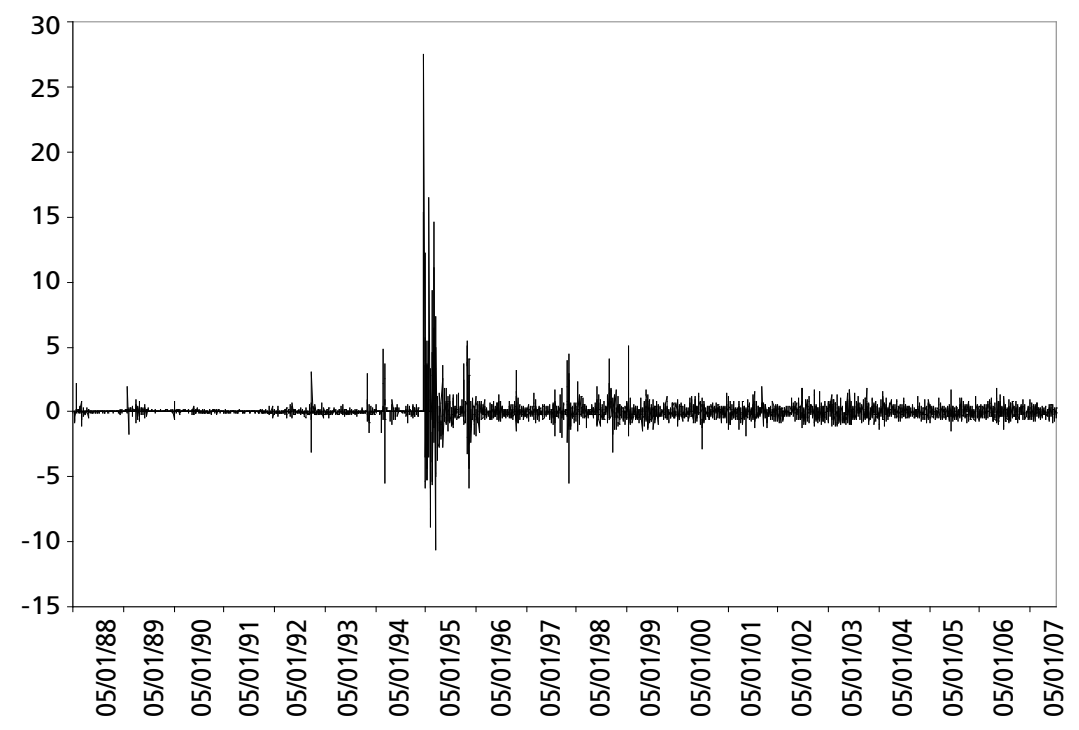

GráfICA 3. Rendimientos diarios del IPC

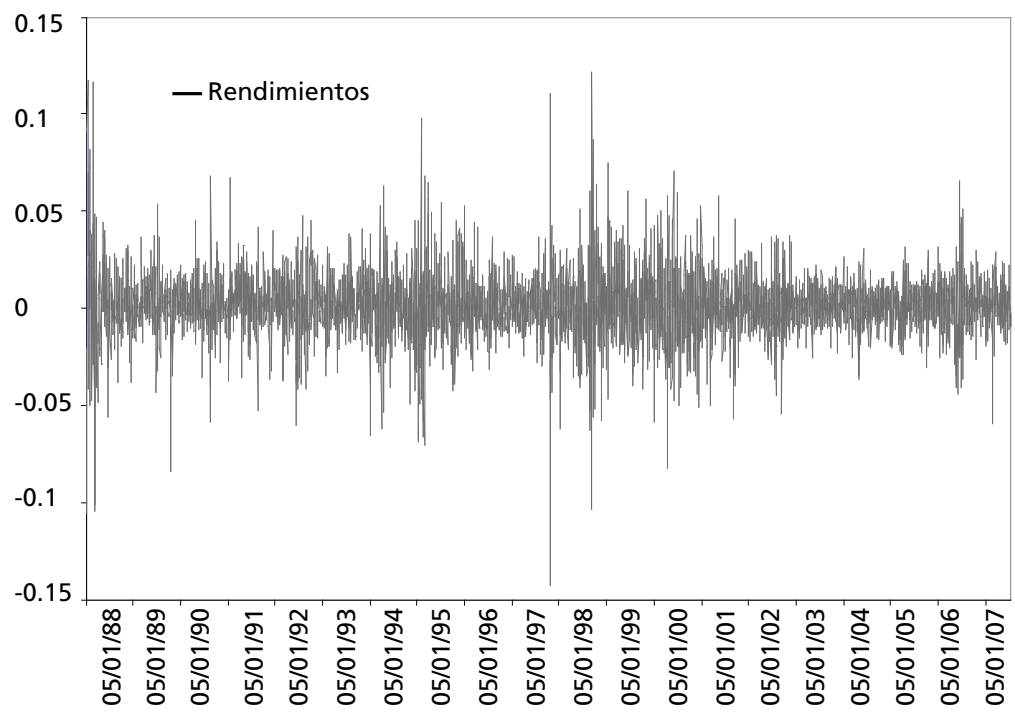

Las gráficas 2 y 3 muestran las variaciones diarias porcentuales del tipo de cambio y los rendimientos diarios del IPC, respectivamente. Como se puede apreciar en la gráfica 3, la varianza de los rendimientos no es constante a lo largo 
del tiempo, sugiriendo que su modelación tome en cuenta alguna forma de heterocedasticidad autorregresiva.

\section{Metodología Y PRINCIPALES RESUltAdOS EMPÍRICOS}

Antes de llevar a cabo la metodología propuesta por Siourounis (2004) para examinar la posible incidencia de las depreciaciones cambiarias en distintas magnitudes sobre los rendimientos accionarios, es necesario verificar la posible existencia de una relación de largo plazo entre las variables.

De acuerdo con Qayyum y Kemal (2006), la especificación para investigar una posible relación de largo plazo entre el mercado accionario, representado por el IPC, y el mercado cambiario, representado por el tipo de cambio fix, es la siguiente:

$$
\operatorname{lip} c_{t}=\alpha+\beta l t c_{t}+\varepsilon_{t}
$$

donde ambas variables están en logaritmos, mientras que la prueba de Engle y Granger se especifica de la siguiente manera:

$$
\Delta \varepsilon_{t}=\beta_{0}+\rho \varepsilon_{t-1}+\beta_{1} \Delta \varepsilon_{t-1}+\beta_{2} \Delta \varepsilon_{t-2}+\ldots+\beta_{k} \Delta \varepsilon_{t-k}+u_{t}
$$

donde $\varepsilon_{t}$ son los residuos de la ecuación cointegrante, establecida en (4), y $u_{t}$ son los residuos de la prueba de la raíz unitaria ADF, los cuales se asumen como ruido blanco.

Los resultados de la estimación son los siguientes:

$$
\begin{gathered}
\quad l i p c_{t}=\underset{(176.16)}{4.5285}+\underset{(144.57)}{1.9473 l^{2 t c} c_{t}} \\
R^{2}=0.8102 \quad F=20900.34 \quad D W=0.0018 \quad A D F=-2.2916(-4.5210)
\end{gathered}
$$

A pesar de lo significativo de los parámetros y del valor relativamente alto del coeficiente de determinación, de $81.02 \%$, el estadístico Durbin-Watson es muy bajo, revelando la presencia de autocorrelación en la estimación, y menor que la $R^{2}$, lo que sugiere que ambas variables no cointegran. Con el fin de realizar una prueba formal de cointegración entre estas series se llevó a cabo la prueba de Engle y Granger, para verificar así la estacionariedad de los residuos de la estimación anterior. 
El valor del estadístico Dickey-Fuller resultó ser de -2.2916, muy por debajo en valor absoluto del valor crítico de $95 \%$ de confianza, lo cual sugiere que ambas series no cointegran; la prueba se especificó sin intercepto y el número de rezagos se eligió de acuerdo con la metodología de lo general a lo particular. Sin embargo, una de las principales limitaciones del método de Engle y Granger para probar cointegración es que asume que el modelo estimado está correctamente especificado, de tal manera que la dirección de la causalidad implícita entre las variables es la correcta.

No obstante lo anterior, un método para probar cointegración entre las series que supera la anterior limitación es la técnica de Johansen, la cual consiste en probar el rango de $\Gamma_{k}$, la matriz de parámetros asociada con el vector de rezagos en los niveles de las variables. Esta técnica especifica el modelo de corrección del error (MCE) de un VAR de $m$-variables para un vector de series de tiempo $X_{t}$ como:

$$
\Delta X_{t}=\Gamma_{1} \Delta X_{t-1}+\Gamma_{2} \Delta X_{t-2} \cdots+\Gamma_{k-1} \Delta X_{t-k+1}+\Gamma_{k} X_{t-k}+v_{t}
$$

donde $\Gamma_{k}$ define la "solución en niveles" de largo plazo en la ecuación (7), Cuthbertson et al. (1992), y $k$ es lo suficientemente grande para asegurar que $v_{t}$ sea un vector de ruido blanco gaussiano que se distribuye idéntica e independientemente con media cero y varianza finita. De esta forma, la técnica prueba el rango de $\Gamma_{k}$, la matriz de parámetros asociada con el vector de rezagos en los niveles de las variables. La longitud de los rezagos fue elegida de acuerdo con el Criterio de Schwarz. Los resultados de la prueba con el método de Johansen se muestran en el cuadro 2.

CuADro 2. Resultados de la prueba de la traza de Johansen

\begin{tabular}{cccccc}
\hline $\begin{array}{c}H_{0}: \text { rango } \\
=p\end{array}$ & Eigenvalor & $-T 1 n\left(1-\hat{\lambda}_{r+1}\right)$ & $95 \%$ & $99 \%$ & Rezagos \\
\hline \multicolumn{5}{c}{ Prueba con } & intercepto en la ecuación cointegrante \\
\hline$p=0$ & 0.0023 & 13.9731 & 15.41 & 20.04 & 4 \\
$p \leq 1$ & 0.0006 & 2.7502 & 3.76 & 6.65 & \\
\hline \multicolumn{5}{c}{ Prueba con intercepto } & y tendencia en la ecuación cointegrante \\
\hline$p=0$ & 0.0040 & 22.5280 & 25.32 & 30.45 & 4 \\
$p \leq 1$ & 0.0006 & 3.0213 & 12.25 & 16.26 & \\
\hline
\end{tabular}

** Indica el rechazo de la hipótesis nula a 1\% de significancia. Pruebas realizadas en Eviews 5.

De forma similar al resultado encontrado por la prueba de Engle y Granger, la prueba de la traza de Johansen tampoco revela la existencia de cointegra- 
ción entre el índice de precios y cotizaciones y el tipo de cambio, ambas variables en logaritmos. Por tanto, de acuerdo con los resultados de ambas pruebas de cointegración, es posible inferir que no se encuentra ninguna relación de largo plazo entre estas variables. Este resultado es consistente con lo encontrado por Qayyum y Kemal (2006) en el caso de Pakistán.

No obstante lo anterior, se llevó a cabo la prueba de causalidad en el sentido de Granger, la cual consiste en estimar un VAR de la forma:

$$
\begin{aligned}
& y_{t}=\alpha_{10}+\alpha_{11} y_{t-1}+\ldots+\alpha_{11} y_{t-l}+\beta_{11} x_{t-1}+\ldots+\beta_{1 l} x_{t-l}+\varepsilon_{t} \\
& x_{t}=\alpha_{20}+\alpha_{21} x_{t-1}+\ldots+\alpha_{2 l} x_{t-l}+\beta_{21} y_{t-1}+\ldots+\beta_{2 l} y_{t-l}+u_{t}
\end{aligned}
$$

y en realizar la siguiente prueba de hipótesis:

$$
\beta_{i 1}=\beta_{i 2}=\ldots=\beta_{i l}=0 .
$$

Dicha hipótesis conjunta se lleva a cabo, para cada ecuación, a partir del estadístico de Wald, el cual se distribuye como una $F$. De esta manera, la hipótesis nula es que $x$ no causa en el sentido de Granger a $y$ en la primera ecuación, y que $y$ no causa en el sentido de Granger a $x$, en la segunda.

Los resultados de la prueba de causalidad de Granger se presentan en el cuadro 3:

CUADRo 3. Resultados de la prueba de causalidad de Granger

\begin{tabular}{ccc}
\hline$H_{0}$ : & Estadístico $F$ & Valor-p \\
\hline lipc no causa en el sentido de Granger a Itc & 40.4105 & 0.0000 \\
Itc no causa en el sentido de Granger a lipc & 3.47615 & 0.0077 \\
\hline
\end{tabular}
Eviews 5.

** Indica el rechazo de la hipótesis nula a $1 \%$ de significancia. Pruebas realizadas en

La prueba de causalidad de Granger, especificada con cuatro rezagos, sugiere que la dirección de la causalidad va en ambos sentidos, es decir, ambas variables se causan simultáneamente en el sentido de Granger. Este resultado cuestiona la dirección de la causalidad entre estas variables establecida en (6), la cual se emplea con frecuencia en la bibliografía empírica sobre el tema. No obstante, estos resultados no imposibilitan el hecho de que en subperiodos el índice de precios y cotizaciones y el tipo de cambio cointegren, o bien que la causalidad 
entre estas variables sea unidireccional o que cambie con el tiempo, como lo reportan Guzmán Plata et al. (2007).

A la luz de estos resultados, se justifica en alguna medida la metodología que emplearemos para examinar la posible incidencia de las depreciaciones cambiarias en los rendimientos accionarios.

De esta forma, cuando se modelan rendimientos diarios se debe tomar en cuenta la posibilidad de que la varianza en el tiempo no sea constante. Asumiendo que $d_{t+1}=0$ y $\rho=0$ es posible estimar el modelo:

$$
P_{t}=\left(E_{t} P_{t+1}+d_{t+1}\right) /(1+\rho)
$$

Y $\sigma_{t}^{2}=\operatorname{Var}\left(R_{t} / I_{t-1}\right)$, donde $E\left(\varepsilon_{t}\right)=0$ es un término de error no autocorrelacionado serialmente.

La varianza del modelo puede ser dependiente del tiempo y expresar se como una función de realizaciones pasadas. Bollerslev (1983) generalizó la metodología ARCH que Engle (1982) introdujo en la bibliografía econométrica. Este tipo de modelo fue encontrado extremadamente útil (Chou, 1988 y French $e t$ $a l ., 1987)$ en economía y finanzas debido a su gran flexibilidad en la modelación de su segundo momento. Si el término del proceso de error es:

$$
\begin{gathered}
\varepsilon_{t}=v_{t} \sqrt{h_{t}} \text {, donde } \sigma_{v}^{2}=1, E\left(v_{t}\right)=0 \\
\mathrm{y} \\
h_{t}=\alpha_{0}+\sum_{i=1}^{q} \alpha_{i} \varepsilon_{t-i}^{2}+\sum_{i=1}^{p} \beta_{i} h_{t-i},
\end{gathered}
$$

entonces la secuencia $\left\{v_{t}\right\}$ es un proceso de ruido blanco y las medias condicional e incondicional de $\varepsilon_{t}$ son iguales a cero.

La media de la serie de rendimientos fue modelada simplemente por el modelo ARMA $(p, q)$ que mejor se ajustó, en este caso viene dado por un AR (2): ${ }^{3}$

$$
R_{t}=b_{0}+b_{1} R_{t-1}+b_{2} R_{t-2}+\varepsilon_{t}
$$

y la varianza muestral para los errores estimados $\varepsilon_{t}^{*}$ obtenidos, está dada por:

$$
\sigma^{2^{*}}=\sum_{t=1}^{T} \varepsilon_{t}^{* 2} / T
$$

${ }^{3}$ Ver cuadro A1 del apéndice A. Cabe aclarar que los residuos de los distintos modelos ARMA estimados para la media en ningún caso resultaron ser ruido blanco. 
donde $T$ es el número de observaciones. Los coeficientes de autocorrelación se calculan a partir de:

$$
\rho_{i}=\sum_{t=i+1}^{T}\left(\varepsilon_{t}^{* 2}-\sigma^{* 2}\right)\left(\varepsilon_{t}^{* 2}-\sigma^{* 2}\right) / \sum_{t=1}^{T}\left(\varepsilon_{t}^{* 2}-\sigma^{* 2}\right)^{2}
$$

El correlograma de los coeficientes de autocorrelación (FAC) y de autocorrelación parcial (FACP) de los residuos al cuadrado del modelo AR(2) se presenta en la gráfica 5. El estadístico $Q$ de Ljung-Box también se calculó para los residuales al cuadrado; el cuadro 4 reporta los valores de la prueba del estadístico $Q$ hasta 24 rezagos. El estadístico $Q$ está dado por:

$$
Q=T(T+2) \sum_{i=1}^{n} \rho(i) /(T-i) .
$$

Y tiene una distribución asintótica $\chi^{2} \operatorname{con}(n-p-q)$ grados de libertad.

CUADRo 4. Diagnósticos de los residuos al cuadrado.

\begin{tabular}{|l|c|}
\hline Estadístico & Residuos al cuadrado \\
\hline Media & 0.00026149 \\
& $(1.091477 \mathrm{e}-05)$ \\
Asimetría & $11.83235^{*}$ \\
Curtosis & $213.86330^{*}$ \\
Ljung-Box Q-Statitics & \\
$Q(4)=$ & $756.2845^{*}$ \\
$Q(8)=$ & $1039.9400^{*}$ \\
$Q(12)=$ & $1304.6632^{*}$ \\
$Q(16)=$ & $1449.7523^{*}$ \\
$Q(20)=$ & $1585.3898^{*}$ \\
$Q(24)=$ & $1667.6065^{*}$ \\
\hline
\end{tabular}

*Denota significancia en el nivel de $1 \%$. En paréntesis se reportan los errores estándar. El periodo que comprende la muestra va del 4 de enero de 1988 al 18 de julio de 2007, para un total de 4,897 observaciones.

Si $\varepsilon_{t}$ depende de realizaciones pasadas y varianzas condicionales pasadas, entonces está dado por (Enders, 2004):

$$
\varepsilon_{t}=v_{t}\left(\alpha_{0}+\sum_{i=1}^{q} \alpha_{i} \varepsilon_{t-i}^{2}+\sum_{i=1}^{p} \beta_{i} h_{t-i}\right)
$$

donde $v_{t} \sim \operatorname{iid}(0,1)$. 
Tal que la varianza condicional de $\varepsilon_{t}$ es:

$$
h_{t}=\alpha_{0}+\sum_{i=1}^{q} \alpha_{i} \varepsilon_{t-i}^{2}+\sum_{i=1}^{p} \beta_{i} h_{t-i},
$$

donde cada realización de $\varepsilon_{t}$ tiene la varianza $h_{t}$, la función de verosimilitud de la observación $t$ (bajo el supuesto de normalidad) es:

$$
-1 / 2 \ln (2 \pi)-1 / 2 \ln h_{t}-1 / 2 h_{t}\left(R_{t}-b R_{t-1}\right)^{2} .
$$

Si se efectúa sobre todas las observaciones disponibles, la función apropiada de verosimilitud es:

$$
\log \vartheta=(-T-1) / 2 \ln (2 \pi)-1 / 2 \ln \sum_{t=2}^{T} h_{t},
$$

donde $h_{t}$ está dado por la ecuación (15).

GRÁFICA 4. Funciones de autocorrelación y de autocorrelación parcial de rendimientos del IPC

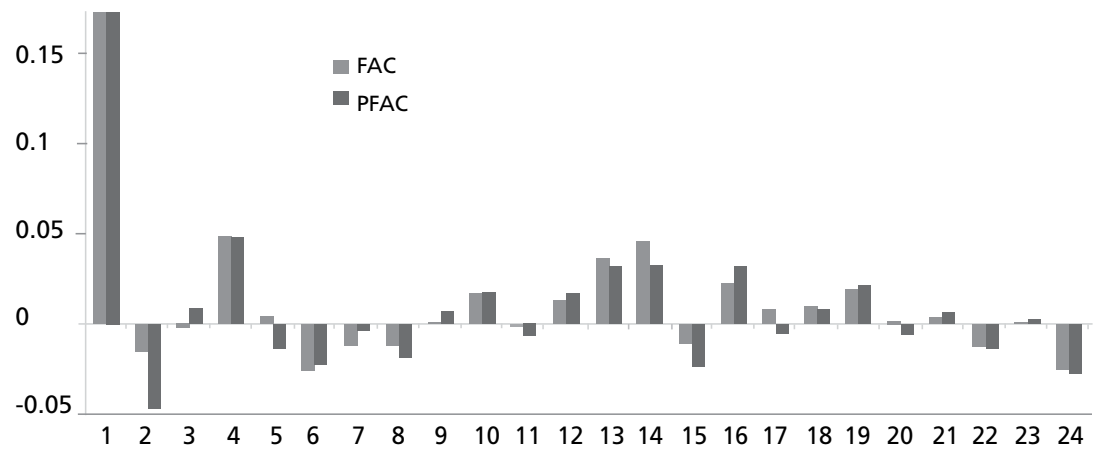

GRÁFICA 5. Funciones de autocorrelación y de autocorrelación parcial de los residuos de la media al cuadrado

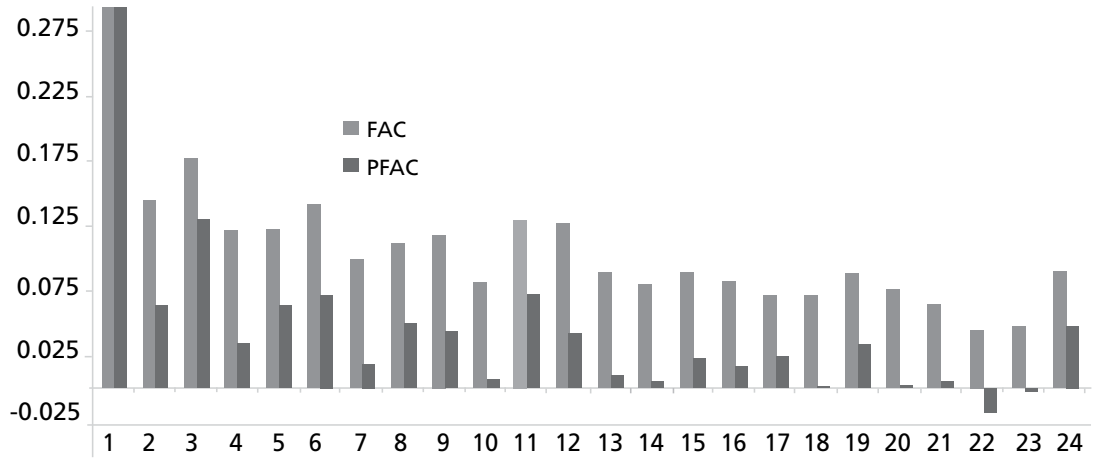


Las FAC y FACP sugieren modelar la varianza de la volatilidad con un orden bajo de rezagos tanto en la parte autorregresiva como en la condicional heterocedástica.

La técnica de máxima verosimilitud proporciona estimaciones para las $b$ 's, $\alpha$ 's y $\beta$ 's en un solo proceso de maximización, empleando un algoritmo de búsqueda. Una especificación con $p=1$ y $q=1$ para la varianza condicional resultó ser óptima.

Los resultados de las estimaciones para el modelo GARCH $(1,1)$ se presentan en el cuadro 5:

CUADRO 5. Estimaciones de máxima verosimilitud del modelo GARCH

\begin{tabular}{ccccccc}
\hline \multicolumn{3}{c}{ Ecuación de la media } & \multicolumn{3}{c}{ Ecuación de la varianza } \\
\hline & $b_{0}$ & $b_{1}$ & $b_{2}$ & $\alpha_{0}$ & $\alpha_{1}$ & $\beta_{1}$ \\
\hline Coeficiente & 0.00142 & 0.19740 & -0.05300 & 0.00001 & 0.13350 & 0.82390 \\
E. S. & $(0.00019)$ & $(0.01500)$ & $(0.01500)$ & $(0.00001)$ & $(0.00638)$ & $(0.00834)$ \\
\hline Observaciones empleadas & \multicolumn{2}{c}{4893} & \multicolumn{2}{c}{ Pruebas de sesgo en el signo* } \\
Grados de libertad & \multicolumn{2}{c}{4887} & En el signo & 0.0892 \\
Valor de la función LM & \multicolumn{2}{c}{13832.45} & Positivo & 0.1323 \\
Asimetría** & \multicolumn{2}{c}{-0.28411} & Negativo & 0.8898 \\
Curtosis** & 6.37101 & Conjunta & & 0.0021 \\
\hline
\end{tabular}

* $y$ ** denotan no significancia de los parámetros estimados en los niveles de $1 \%$ y $5 \%$, respectivamente. Los números entre paréntesis son los errores estándar. El periodo que comprende la muestra va del 4 de enero de 1988 al 18 de julio del 2007, para un total de 4,896 observaciones. El modelo estimado es: $R_{t}=b_{0}+b_{1} R_{t-1}+b_{2} R_{t-2}+\varepsilon_{t}$ donde $\varepsilon_{t}=v_{t} \sqrt{h_{t}}$ y $h_{t}=\alpha_{0}+\alpha_{1} \varepsilon_{t-1}^{2}+\beta_{1} h_{t-1}$.

* Probabilidades de la prueba. **De los residuos normalizados.

La gráfica 6 muestra la raíz cuadrada de la varianza condicional estimada del modelo GARCH $(1,1)$ :

GRÁFICA 6. La raíz cuadrada de la varianza condicional estimada del modelo GARCH $(1,1)$.

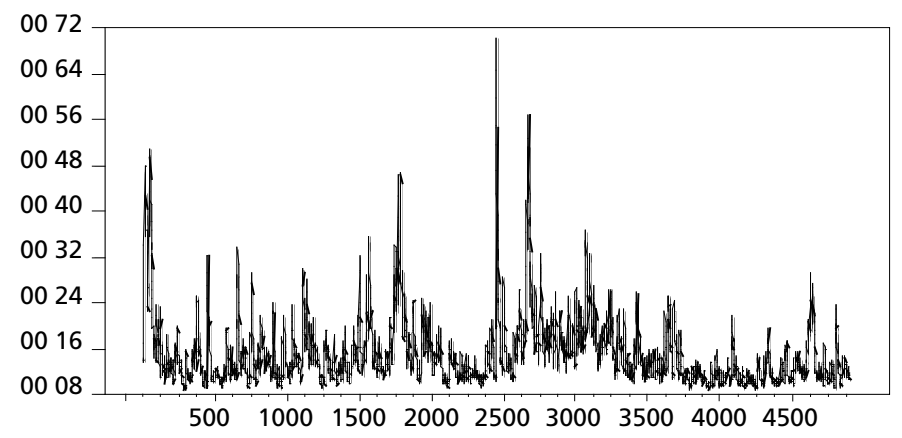


Con la finalidad de evaluar el ajuste y la capacidad de los modelos para modelar la varianza, en el cuadro 6 se presenta un diagnóstico de los residuos al cuadrado normalizados:

CUADRo 6. Diagnóstico en los residuos al cuadrado normalizados.

\begin{tabular}{|lc|}
\hline Estadístico & GARCH $(1,1)$ \\
\hline Media & 1.0009 \\
& $(2.326867)$ \\
Varianza & 5.4143 \\
Asimetría & $14.58177 * *$ \\
Curtosis & $414.9875^{* *}$ \\
Prueba de Q-Ljung-Box para correlación serial en residuos al cuadrado \\
LB(4) \\
LB(8) \\
LB(12) \\
LB(16) \\
LB(20) \\
LB(24) \\
\hline
\end{tabular}

${ }^{*} y * *$ denotan significancia en los niveles del 5 y $1 \%$ respectivamente.

Los resultados del diagnóstico muestran que los valores del estadístico $Q$-Ljung-Box del modelo GARCH $(1,1)$ resultaron no significativos en los niveles convencionales, con excepción del LB (4), que es significativo a $5 \%$ pero no a $1 \%$; de aquí es posible concluir que los residuos al cuadrado estandarizados no están serialmente correlacionados y que por tanto el modelo ajusta adecuadamente la volatilidad de los rendimientos del IPC.

Del cuadro 5 se puede ver que todos los coeficientes estimados del modelo GARCH $(1,1)$ resultaron significativos al nivel de significancia de $1 \%$. Sin embargo, la evidencia señala que este modelo no capta totalmente los impactos en la volatilidad, ya que al aplicar las pruebas de diagnóstico para los modelos de volatilidad, sugeridas por Engle y $\mathrm{Ng}$ (1993), que examinan la posibilidad de pronosticar los residuos al cuadrado normalizados por algunas variables observadas no incorporadas en el modelo, la prueba del sesgo en el signo de manera conjunta sugiere incorporar un efecto asimétrico para la varianza. Mientras que el modelo LGARCH $(1,1)$, además de tener todos los parámetros significativos, capta 
totalmente los impactos en la volatilidad de los choques positivos y negativos tanto de manera individual como en forma conjunta (Hernández et al. (2007). No obstante lo anterior, para los fines de este trabajo, empleamos un modelo GARCH $(1,1)$ con variables exógenas para probar la hipótesis planteada.

De esta forma, con el fin de averiguar si la ecuación para la media y/o la varianza condicional es afectada por las depreciaciones del tipo de cambio, una variable dummy es incorporada en las ecuaciones (8) y (14): ${ }^{3}$

$$
\begin{aligned}
& R_{t}=b_{0}+b_{1} R_{t-1}+b_{2} R_{t-2}+\varepsilon_{t}+\varphi_{m} D_{t} \\
& \varepsilon_{t}=v_{t} \sqrt{h_{t}} \\
& h_{t}=\alpha_{0}+\alpha_{1} \varepsilon_{t-1}^{2}+\beta_{1} h_{t-1}+\varphi_{v} D_{t}
\end{aligned}
$$

Donde la variable dummy, $D_{t}$, da cuenta de si la depreciación del tipo de cambio $^{4}$ es mayor que un determinado umbral, como se describe a continuación:

$$
D_{t}=\left\{\begin{array}{l}
1, \text { si el incremento porcentual de e } e s \geq \text { Umbral } \\
0, \text { de otraforma }
\end{array}\right.
$$

Véase los resultados de las estimaciones para el caso de las depreciaciones en el cuadro 7:

\section{CUADRo 7. Estimaciones de máxima verosimilitud del modelo GARCH $(1,1)$ con depreciaciones (continuación)}

\begin{tabular}{ccccccccc}
\hline & \multicolumn{4}{c}{ Ecuación de la media } & \multicolumn{5}{c}{ Ecuación de la varianza } \\
\hline$\Delta \mathrm{e}^{* * *}$ & $b_{0}$ & $b_{1}$ & $b_{2}$ & $\phi_{\mathrm{m}}$ & $\alpha_{0}$ & $\alpha_{1}$ & $\beta_{1}$ & $\phi_{\mathrm{v}}$ \\
\hline & 0.00181 & 0.19580 & -0.05670 & -0.00099 & 0.00001 & 0.13250 & 0.82450 & \\
& $(0.00024)$ & $(0.01500)$ & $(0.01520)$ & $(0.00037)$ & $(0.00000)$ & $(0.00643)$ & $(0.00839)$ & \\
$0.05 \%$ & 0.00142 & 0.19760 & -0.05310 & & 0.00001 & 0.13420 & 0.82280 & $0.00000 * *$ \\
& $(0.00019)$ & $(0.01500)$ & $(0.01500)$ & & $(0.00000)$ & $(0.00644)$ & $(0.00858)$ & $(0.00000)$ \\
& 0.00181 & 0.19610 & -0.05680 & -0.00099 & 0.00001 & 0.13330 & 0.82330 & $0.00000 * *$ \\
& $(0.00024)$ & $(0.01500)$ & $(0.01520)$ & $(0.00037)$ & $(0.00000)$ & $(0.00649)$ & $(0.00864)$ & $(0.00000)$ \\
\hline & 0.00188 & 0.19460 & -0.05790 & -0.00139 & 0.00001 & 0.13250 & 0.82510 & \\
& $(0.00023)$ & $(0.01500)$ & $(0.01510)$ & $(0.00039)$ & $(0.00000)$ & $(0.00646)$ & $(0.00842)$ & \\
$0.10 \%$ & 0.00142 & 0.19740 & -0.05300 & & 0.00001 & 0.13340 & 0.82400 & $0.00000 * *$ \\
& $(0.00019)$ & $(0.01510)$ & $(0.01500)$ & & $(0.00000)$ & $(0.00645)$ & $(0.00846)$ & $(0.00000)$ \\
& 0.00188 & 0.19460 & -0.05790 & -0.00139 & 0.00001 & 0.13250 & 0.82510 & $0.00000 * *$ \\
& $(0.00023)$ & $(0.01500)$ & $(0.01510)$ & $(0.00039)$ & $(0.00000)$ & $(0.00653)$ & $(0.00854)$ & $(0.00000)$ \\
\hline
\end{tabular}

\footnotetext{
${ }^{3}$ El paquete econométrico RATS cuenta con rutinas para estimar distintos modelos de la familia GARCH que permiten incorporar variables exógenas tanto en la ecuación de la media como en la de la varianza o en ambas.

${ }^{4}$ Es importante destacar que lo que denominamos depreciación del tipo de cambio son variaciones porcentuales del tipo de cambio, calculadas de la siguiente forma: $\Delta e \%=\left[\left(e_{t}-e_{t-1}\right) / e_{t-1}\right] \times 100$.
} 


\section{CUADRo 7. Estimaciones de máxima verosimilitud del modelo GARCH $(1,1)$ con depreciaciones \\ (continuación)}

\begin{tabular}{|c|c|c|c|c|c|c|c|c|}
\hline \multirow[b]{2}{*}{$\Delta \mathrm{e}^{* * * *}$} & \multicolumn{4}{|c|}{ Ecuación de la media } & \multicolumn{4}{|c|}{ Ecuación de la varianza } \\
\hline & $b_{0}$ & $b_{1}$ & $b_{2}$ & $\phi_{m}$ & $\alpha_{0}$ & $\alpha_{1}$ & $\beta_{1}$ & $\phi_{v}$ \\
\hline \multirow{6}{*}{$0.15 \%$} & 0.00182 & 0.19460 & -0.05770 & -0.00143 & 0.00001 & 0.13160 & 0.82640 & \\
\hline & $(0.00022)$ & $(0.01500)$ & $(0.01510)$ & $(0.00042)$ & $(0.00000)$ & $(0.00644)$ & $(0.00837)$ & \\
\hline & 0.00144 & 0.19670 & -0.05270 & & 0.00001 & 0.13340 & 0.82710 & 0.00000 \\
\hline & $(0.00019)$ & $(0.01510)$ & $(0.01500)$ & & $(0.00000)$ & $(0.00665)$ & $(0.00824)$ & $(0.00000)$ \\
\hline & 0.00183 & 0.19400 & -0.05740 & -0.00141 & 0.00001 & 0.13160 & 0.82940 & 0.00000 \\
\hline & $(0.00023)$ & $(0.01510)$ & $(0.01510)$ & $(0.00041)$ & $(0.00000)$ & $(0.00670)$ & $(0.00828)$ & $(0.00000)$ \\
\hline \multirow{6}{*}{$0.20 \%$} & 0.00180 & 0.19410 & -0.05860 & -0.00157 & 0.00001 & 0.13130 & 0.82670 & \\
\hline & $(0.00022)$ & $(0.01500)$ & $(0.01510)$ & $(0.00043)$ & $(0.00000)$ & $(0.00640)$ & $(0.00833)$ & \\
\hline & 0.00143 & 0.19670 & -0.05280 & & 0.00001 & 0.13340 & 0.82640 & $0.00000^{*}$ \\
\hline & $(0.00019)$ & $(0.01510)$ & $(0.01500)$ & & $(0.00000)$ & $(0.00661)$ & $(0.00827)$ & $(0.00000)$ \\
\hline & 0.00180 & 0.19410 & -0.05860 & -0.00157 & 0.00001 & 0.13130 & 0.82670 & $0.00000^{*}$ \\
\hline & $(0.00022)$ & $(0.01500)$ & $(0.01510)$ & $(0.00043)$ & $(0.00000)$ & $(0.00640)$ & $(0.00833)$ & $(0.00000)$ \\
\hline \multirow{6}{*}{$25 \%$} & 0.00176 & 0.19470 & -0.0 & -0.00162 & 0.00001 & 0.13100 & 0.82720 & \\
\hline & $(0.00021)$ & $(0.01500)$ & $(0.01510)$ & $(0.00045)$ & $(0.00000)$ & $(0.00637)$ & $(0.00831)$ & \\
\hline & 0.00143 & 0.19710 & -0.05290 & & 0.00001 & 0.13320 & 0.82540 & $0.00000 * *$ \\
\hline & $(0.00019)$ & $(0.01510)$ & $(0.01500)$ & & $(0.00000)$ & $(0.00656)$ & $(0.00828)$ & $(0.00000)$ \\
\hline & 0.00176 & 0.19440 & -0.05870 & -0.00161 & 0.00001 & 0.13 & 0.82860 & $0.00000 * *$ \\
\hline & $(0.00021)$ & $(0.01500)$ & $(0.01510)$ & $(0.00044)$ & $(0.00000)$ & $(0.00653)$ & $(0.00826)$ & $(0.00000)$ \\
\hline \multirow{6}{*}{$0.30 \%$} & 0.00176 & 90 & -0.0 & -0.00193 & 0.00001 & 0.13120 & 0.82670 & \\
\hline & $(0.00021)$ & $(0.01500)$ & $(0.01520)$ & $(0.00048)$ & $(0.00$ & $(0.00640)$ & $(0.0$ & \\
\hline & 0.00142 & 0.19760 & -0.05310 & & 0.00001 & 0.13370 & 0.82290 & $0.00000^{* *}$ \\
\hline & (0.00019) & $(0.01510)$ & $(0.01500)$ & & $(0.00000)$ & $(0.00658)$ & $(0.00838)$ & $(0.00000)$ \\
\hline & 0.00175 & 0.19520 & -0.06010 & -0.00194 & 0.00001 & 0.13150 & 0.82560 & $0.00000^{* *}$ \\
\hline & $(0.00021)$ & $(0.01500)$ & $(0.01520)$ & $(0.00048)$ & $(0.00000)$ & $(0.00657)$ & $(0.00841)$ & $(0.00000)$ \\
\hline \multirow{6}{*}{$0.40 \%$} & 0.00164 & 0.19570 & -0.05800 & -0.00168 & 0.00001 & 0.13300 & 0.82480 & \\
\hline & $(0.00020)$ & $(0.01510)$ & $(0.01510)$ & $(0.00054)$ & $(0.00000)$ & $(0.00650)$ & $(0.00845)$ & \\
\hline & 0.00142 & 0.19740 & -0.05300 & & 0.00 & 0.13 & 0.82400 & $0.00000^{* *}$ \\
\hline & $(0.00019)$ & $(0.01500)$ & $(0.01500)$ & & $(0.00000)$ & $(0.00654)$ & $(0.00834)$ & $(0.00000)$ \\
\hline & 0.00165 & 0.19560 & -0.05800 & -0.00168 & 0.00001 & 0.13300 & 0.82510 & $0.00000^{* *}$ \\
\hline & $(0.00020)$ & $(0.01510)$ & $(0.01510)$ & $(0.00054)$ & $(0.00000)$ & $(0.00662)$ & $(0.00845)$ & $(0.00000)$ \\
\hline \multirow{6}{*}{$0.50 \%$} & 0.00156 & 9630 & -0.05620 & $-0.00149 *$ & 0.00001 & 0.13320 & 0.82440 & \\
\hline & $(0.00020)$ & $(0.01500)$ & $(0.01510)$ & $(0.00061)$ & $(0.00000)$ & $(0.00650)$ & $(0.00847)$ & \\
\hline & 0.00142 & 0.19850 & -0.05300 & & 0.00001 & 0.13450 & 0.81880 & $0.00001 *$ \\
\hline & $(0.00019)$ & $(0.01510)$ & $(0.01500)$ & & $(0.00000)$ & $(0.00668)$ & $(0.00862)$ & $(0.00000)$ \\
\hline & 0.00155 & 0.19730 & -0.05610 & -0.00150 & 0.00001 & 0.13410 & 0.81950 & $0.00001 *$ \\
\hline & $(0.00020)$ & $(0.01510)$ & $(0.01520)$ & $(0.00065)$ & $(0.00000)$ & $(0.00675)$ & $(0.00874)$ & $(0.00000)$ \\
\hline \multirow{6}{*}{$0.75 \%$} & 0.00145 & 0.19700 & -0.05380 & $-0.00068^{\star *}$ & 0.00001 & 0.13340 & 0.82400 & \\
\hline & $(0.00020)$ & $(0.01510)$ & $(0.01510)$ & $(0.00086)$ & $(0.00000)$ & (0.00638) & $(0.00834)$ & \\
\hline & 0.00142 & 0.19850 & -0.05310 & & 0.00001 & 0.13470 & 0.81920 & $0.00001^{* *}$ \\
\hline & $(0.00019)$ & $(0.01510)$ & $(0.01500)$ & & $(0.00000)$ & (0.00645) & $(0.00898)$ & $(0.0$ \\
\hline & $0.0 c$ & 0.19810 & -0.05390 & & 0.00001 & 0.13460 & 0.81930 & $0.00001 * \star$ \\
\hline & $(0.00020)$ & $(0.01510)$ & $(0.01510)$ & $(0.00092)$ & $(0.00000)$ & $(0.00645)$ & $(0.00898)$ & $(0.00001)$ \\
\hline
\end{tabular}




\section{CUADRO 7. Estimaciones de máxima verosimilitud del modelo GARCH $(1,1)$ con depreciaciones \\ (conclusión)}

\begin{tabular}{|c|c|c|c|c|c|c|c|c|}
\hline \multirow[b]{2}{*}{$\Delta \mathrm{e}^{* * *}$} & \multicolumn{4}{|c|}{ Ecuación de la media } & \multicolumn{4}{|c|}{ Ecuación de la varianza } \\
\hline & $b_{0}$ & $b_{1}$ & $b_{2}$ & $\phi_{m}$ & $\alpha_{0}$ & $\alpha_{1}$ & $\beta_{1}$ & $\phi_{\mathrm{v}}$ \\
\hline \multirow{6}{*}{$1.00 \%$} & 0.00142 & 0.19740 & -0.05280 & $0.00031 * *$ & 0.00001 & 0.13350 & 0.82400 & \\
\hline & $(0.00020)$ & $(0.01510)$ & $(0.01510)$ & $(0.00115)$ & $(0.00000)$ & $(0.00638)$ & $(0.00833)$ & \\
\hline & 0.00141 & 0.19990 & -0.05340 & & 0.00001 & 0.13680 & 0.81180 & 0.00003 \\
\hline & $(0.00019)$ & $(0.01520)$ & $(0.01500)$ & & $(0.00000)$ & $(0.00664)$ & $(0.00957)$ & $(0.00001)$ \\
\hline & 0.00141 & 0.19990 & -0.05340 & $0.00006 * *$ & 0.00001 & 0.13680 & 0.81180 & 0.00003 \\
\hline & $(0.00019)$ & $(0.01520)$ & $(0.01510)$ & $(0.00143)$ & $(0.00000)$ & $(0.00665)$ & $(0.00958)$ & $(0.00001)$ \\
\hline \multirow{3}{*}{$1.50 \%$} & $\begin{array}{c}0.00141 \\
(0.00019)\end{array}$ & $\begin{array}{c}0.19720 \\
(0.01510)\end{array}$ & $\begin{array}{l}-0.05240 \\
(0.01510)\end{array}$ & $\begin{array}{l}0.00139 * * \\
(0.00188)\end{array}$ & $\begin{array}{c}0.00001 \\
(0.00000)\end{array}$ & $\begin{array}{c}0.13320 \\
(0.00636)\end{array}$ & $\begin{array}{c}0.82450 \\
(0.00829)\end{array}$ & \\
\hline & $\begin{array}{c}0.00140 \\
(0.00019)\end{array}$ & $\begin{array}{c}0.19910 \\
(0.01520)\end{array}$ & $\begin{array}{l}-0.05380 \\
(0.01500)\end{array}$ & & $\begin{array}{c}0.00001 \\
(0.00000)\end{array}$ & $\begin{array}{c}0.13600 \\
(0.00665)\end{array}$ & $\begin{array}{c}0.81250 \\
(0.00954)\end{array}$ & $\begin{array}{c}0.00006 \\
(0.00002)\end{array}$ \\
\hline & $\begin{array}{c}0.00140 \\
(0.00019)\end{array}$ & $\begin{array}{c}0.19900 \\
(0.01520)\end{array}$ & $\begin{array}{l}-0.05340 \\
(0.01510)\end{array}$ & $\begin{array}{l}0.00095^{* *} \\
(0.00236)\end{array}$ & $\begin{array}{c}0.00001 \\
(0.00000)\end{array}$ & $\begin{array}{c}0.13570 \\
(0.00664)\end{array}$ & $\begin{array}{c}0.81310 \\
(0.00953)\end{array}$ & $\begin{array}{c}0.00006 \\
(0.00002)\end{array}$ \\
\hline \multirow{5}{*}{$2.00 \%$} & $\begin{array}{c}0.00141 \\
(0.00019)\end{array}$ & $\begin{array}{c}0.19740 \\
(0.01510)\end{array}$ & $\begin{array}{c}-0.05260 \\
(0.01510)\end{array}$ & $\begin{array}{l}0.00264^{* *} \\
(0.00233)\end{array}$ & & & $\begin{array}{c}0.82500 \\
(0.00826)\end{array}$ & \\
\hline & 0.00139 & 0.19900 & -0.05320 & & 0.00001 & 0.13520 & 0.80930 & 0.00017 \\
\hline & $(0.00019)$ & $(0.01530)$ & $(0.01500)$ & & $(0.00000)$ & $(0.00663)$ & $(0.00968)$ & $(0.00005)$ \\
\hline & 0.00139 & 0.19900 & -0.05280 & 0.00230 ** & 0.00 & 0.13490 & 0.80990 & 0.00017 \\
\hline & $(0.00019)$ & $(0.01530)$ & $(0.01510)$ & $(0.00353)$ & $(0.000$ & $(0.00661)$ & $(0.00966)$ & $(0.00005)$ \\
\hline \multirow{3}{*}{$2.50 \%$} & $\begin{array}{c}0.00142 \\
(0.00019)\end{array}$ & $\begin{array}{r}0.1 \\
(0.0\end{array}$ & $\begin{array}{l}-0.0 \\
(0.0\end{array}$ & $\begin{array}{l}0.00173 * * \\
(0.00252)\end{array}$ & $\begin{array}{r}0 . \\
(0 .\end{array}$ & & $\begin{array}{l}0 \\
9)\end{array}$ & \\
\hline & $\begin{array}{c}0.00140 \\
(0.00019)\end{array}$ & $\begin{array}{c}0.19860 \\
(0.01530)\end{array}$ & $\begin{array}{l}-0.05350 \\
(0.01500)\end{array}$ & & $\begin{array}{c}0.00001 \\
(0.00000)\end{array}$ & $\begin{array}{c}0.13570 \\
(0.00665)\end{array}$ & $\begin{array}{c}0.80930 \\
(0.00967)\end{array}$ & $\begin{array}{c}0.00018 \\
(0.00005)\end{array}$ \\
\hline & $\begin{array}{c}0.00139 \\
(0.00019) \\
\end{array}$ & $\begin{array}{c}0.19850 \\
(0.01530) \\
\end{array}$ & $\begin{array}{l}-0.05310 \\
(0.01510) \\
\end{array}$ & $\begin{array}{l}0.00177^{* *} \\
(0.00385) \\
\end{array}$ & $\begin{array}{c}0.00001 \\
(0.00000) \\
\end{array}$ & $\begin{array}{c}0.13550 \\
(0.00665) \\
\end{array}$ & $\begin{array}{c}0.80970 \\
(0.00967) \\
\end{array}$ & $\begin{array}{c}0.00018 \\
(0.00005) \\
\end{array}$ \\
\hline \multirow{6}{*}{$3.00 \%$} & 0.00142 & 0.15140 & -0.05270 & $0.00225^{*}$ & 0.0 & & 0.024 & \\
\hline & $(0.00019)$ & $(0.01510)$ & $(0.01510)$ & $(0.00275)$ & $(0.0$ & $(0.00$ & $(0.00828)$ & \\
\hline & 0.00140 & 0.19910 & -0.05310 & & 0.0 & 0.13470 & 0.81050 & 0.00 \\
\hline & $(0.00019)$ & $(0.01530)$ & $(0.01500)$ & & $(0.00000)$ & $(0.00659)$ & $(0.00959)$ & $(0.00006)$ \\
\hline & 0.00139 & 0.19910 & -0.05280 & $0.00217 * *$ & 0.00001 & 0.13450 & 0.81070 & 0.00024 \\
\hline & $(0.00019)$ & $(0.01530)$ & $(0.01510)$ & $(0.00458)$ & $(0.00000)$ & $(0.00659)$ & $(0.00959)$ & $(0.00007)$ \\
\hline \multirow{4}{*}{$3.50 \%$} & $\begin{array}{c}0.00142 \\
(0.00019)\end{array}$ & 9740 & $\begin{array}{c}-0.05290 \\
(0.01510)\end{array}$ & $\begin{array}{l}0.00067 * * \\
(0.00277)\end{array}$ & 0.00001 & 0.13330 & $\begin{array}{c}0.82410 \\
(0.00833)\end{array}$ & \\
\hline & 0.00139 & 0.19900 & -0.05260 & & 0.000 & 0.13400 & 0.81170 & 0.0 \\
\hline & $(0.00019)$ & $(0.01530)$ & $(0.01500)$ & & $(0.00000)$ & $(0.00654)$ & $(0.00943)$ & $(0.00007)$ \\
\hline & $\begin{array}{c}0.00139 \\
(0.00019)\end{array}$ & $\begin{array}{c}0.19900 \\
(0.01530)\end{array}$ & $\begin{array}{l}-0.05240 \\
(0.01510)\end{array}$ & $\begin{array}{l}0.00117^{* *} \\
(0.00501)\end{array}$ & $\begin{array}{c}0.00001 \\
(0.00000)\end{array}$ & $\begin{array}{c}0.13400 \\
(0.00655)\end{array}$ & $\begin{array}{c}0.81180 \\
(0.00944)\end{array}$ & $\begin{array}{c}0.00027 \\
(0.00008)\end{array}$ \\
\hline
\end{tabular}

* $y$ ** denotan no significancia de los parámetros estimados en los niveles de $1 \%$ y de $5 \%$, respectivamente. Los números entre paréntesis son los errores estándar. El periodo que comprende la muestra va del 4 de enero de 1988 al 18 de julio de 2007, para un total de 4,896 observaciones.

$* * * \Delta$ e es la magnitud de la depreciación, en términos porcentuales, como se definió anteriormente. 
Las estimaciones revelaron un resultado interesante: los parámetros correspondientes a la variable dummy, que incorpora la magnitud de las depreciaciones cambiarias cuando éstas superan un determinado valor, resultaron ser significativos únicamente en la ecuación de la media y no en la varianza, para un intervalo de las depreciaciones diarias de entre $0.05 \%$ a $0.50 \%$, con una incidencia negativa en los rendimientos. Una excepción a lo anterior se da cuando las depreciaciones son de $0.15 \%$, en la que los parámetros de la varianza resultaron significativos; no obstante lo anterior, en todos los casos los parámetros estimados para dicha variable en la ecuación de la varianza son prácticamente nulos.

Por lo contrario, cuando la magnitud de las depreciaciones cambiarias va de $1 \%$ a $3.50 \%$, los parámetros correspondientes a la variable dummy que registra dichos eventos son significativos sólo en la ecuación de la varianza y no en la de la media de los rendimientos en las estimaciones, tanto de manera individual como de forma conjunta.

Un caso particular a destacar es el hecho de que los parámetros estimados de la variable dummy para las depreciaciones cambiarias de $0.75 \%$ no resultaron significativos en ninguna de las ecuaciones de media y varianza.

Estos resultados sugieren que cuando la magnitud de las depreciaciones cambiarias son relativamente pequeñas, de entre $0.05 \%$ y $0.50 \%$, éstas sólo inciden en la media de los rendimientos accionarios y no en su volatilidad. En tanto, cuando las depreciaciones cambiarias son mayores de entre $1 \%$ a 3.5\%, éstas tienden sólo a incrementar la volatilidad del mercado accionario y no tienen impacto alguno en los rendimientos promedio.

\section{Conclusiones}

A partir de la flexibilidad que permiten los modelos GARCH para incorporar variables exógenas en las ecuaciones, tanto de la media como de la varianza o en ambas, hemos analizado la posible influencia de las depreciaciones cambiarias de distinta magnitud en los rendimientos accionarios y en su volatilidad. Un modelo GARCH $(1,1)$ fue el que mejor se ajustó a los datos del mercado accionario y fue empleado para captar la influencia de las depreciaciones del tipo de cambio en este mercado. Los resultados indican que la incidencia tanto en los rendimientos promedio del mercado accionario como en su volatilidad depende de la magnitud de la depreciación, en términos porcentuales, del tipo de cambio. Cuando las depreciaciones del tipo de cambio son relativamente pequeñas, menores a $0.50 \%$, éstas sólo impactan en los rendimientos del mercado bursátil y no en su volatilidad, considerada por su varianza, mientras que cuando éstas superan dicho um- 
bral reportan una mayor incidencia en la volatilidad de este mercado y ya no en sus rendimientos promedio.

Estos resultados están sujetos a la metodología empleada con las debidas limitaciones que ésta pudiera tener. Sin embargo, se requiere una mayor investigación para el estudio del vínculo y las interrelaciones que existen entre los mercados accionario y cambiario. Sería interesante también explorar el impacto de las apreciaciones del tipo de cambio en los rendimientos accionarios, con la metodología empleada en este trabajo, así como también la forma en la cual posiblemente reaccionen estos últimos a la magnitud de las variaciones del tipo de cambio, y no sólo a la dirección de la variación del tipo de cambio.

Otra posible línea de investigación en el estudio de la forma en que ambos mercados se encuentran vinculados puede ser la utilización de modelos GARCH multivariados los cuales pueden arrojar luz sobre la manera en que se transmite la volatilidad entre ambos mercados.

ApÉNDice A

CuAdro A1. Estimación de Box-Jenkins

para la ecuación de la media de los rendimientos diarios $\left(r_{1}\right)$

\begin{tabular}{|c|c|c|c|c|c|}
\hline Modelo & Variable & Coeficiente & Error estándar & Estadístico- $t$ & Significancia \\
\hline \multirow[t]{2}{*}{ 1) } & $b_{0}$ & 0.00118 & 0.00028 & 4.214 & $0.000 *$ \\
\hline & $b_{1}$ & 0.17252 & 0.01407 & 12.258 & $0.000 *$ \\
\hline \multicolumn{6}{|c|}{ aic $=1259.88838$ sbc $=1272.88032$} \\
\hline \multirow[t]{3}{*}{ 2) } & $b_{0}$ & 0.00121 & 0.00026 & 4.530 & $0.000 *$ \\
\hline & $b_{1}$ & 0.17764 & 0.01423 & 12.485 & $0.000 *$ \\
\hline & $b_{2}$ & -0.04622 & 0.01422 & -3.250 & $0.001 *$ \\
\hline \multicolumn{6}{|c|}{ aic $=1213.08113 \mathrm{sbc}=1232.56843$} \\
\hline \multirow[t]{2}{*}{ 3) } & $a_{0}$ & 0.00117 & 0.00027 & 4.271 & $0.000 *$ \\
\hline & $a_{1}$ & 0.18265 & 0.01405 & 12.997 & $0.000 *$ \\
\hline \multicolumn{6}{|c|}{ aic $=841.61560 \quad s b c=854.39205$} \\
\hline
\end{tabular}

* Denota significancia en el nivel de 1\%. El periodo muestral comprende del 6 de enero de 1988 al 18 de julio de 2007, para un total de 4,896 observaciones. El modelo estimado para la media es: (1) $R_{t}=b_{0}+b_{1} R_{t-1}$, (2) $R_{t}=b_{0}+b_{1} R_{t-1}+b_{2} R_{t-2}+\varepsilon_{t}$, (3) $R_{t}=a_{0}+a_{1} \varepsilon_{t-1}+\varepsilon_{t}$. Se emplearon los criterios de Akaike (AIC) y de Schwartz en la selección del mejor modelo con las variables significativas. 


\section{REFERENCIAS BIBLIOGRÁFICAS}

Black, F. (1976), "Studies of Stock Price Volatility Changes". Proceedings of the 1976 Business Meeting of the Business and Economics Statistics Section American Statistical Association, pp. 177-181.

Bekaert, Geert, y Wu Guojun (2000), "Asymmetric Volatility and Risk in Equity Market" Review of Financial Studies, vol. 13, No. 1, pp. 1-42.

Bollerslev, T., R. Y. Chou, y K. F. Kroner (1992), “Arch modeling in finance: a review of the theory and empirical evidence, Journal of Econometrics, 8, pp. 231-43.

Brown, Keith C., et al. (1993), "The Risk and Required Return of Common Stock following Major Price Innovations", Journal of Financial and Quantitative Analysis, vol. 28, núm. 1, marzo, pp. 101-116.

Cai, J. (1997), "Glamour and value strategies on the Tokyo stock exchange", Journal of Business Finance and Accounting, 24, 1291.

Céspedes, L., R. Chang, y A. Velasco (2004), "Balance Sheets and Exchange Rate Policy", American Economic Review, American Economic Association, vol. 94 (4), pp. 1183-1193, septiembre.

Chappel, D., J. Padmore, y J. Pidgeon (1998), "A note on ERM membership and the efficiency of the London Stock Exchange, Applied Economics Letters, 5, p. 19.

Chou, R. Y. (1988), "Volatility persistence and stock valuations: some empirical evidence using GARCH", Journal of Applied Econometrics, 3, pp. 279-294.

Cuthbertson, Keith, et al. (1992), Applied Econometric Techniques, The University of Michigan Press.

Diamond, Douglas, y Robert E. Verrecchia (1982), “Optimal managerial contracts and equilibrium security prices”, Journal of Finance, mayo, núm. 37, pp. 275-287.

Dockery, E., y F. Vergari (1997), "Testing the random walk hypothesis evidence for the Budapest stock exchange", Applied Economics Letters, 4, p. 627.

Enders, W. (2005), Applied Econometric Time Series, John Wiley, Nueva York.

Engle, R. F. (1982), “Autoregressive conditional heteroscedasticity with estimates of the variance of United Kingdom's inflation”, Econometrica, 50, pp. 987-1007. y Victor Ng (1993), "Measuring and Testing the Impact of news on Volatility", The Journal of Finance, vol. XLVIII, núm. 5, diciembre.

Erunza, V. R, y E. Losq (1985), "The behavior of stock prices in LDC markets", Journal of Banking and Finance, 9, pp. 561-575.

French, K. R., G. W. Schwert, y R. F. Stambaugh (1987), "Expected returns and volatility", Journal of Financial Economics, 19, pp. 3-29. 
Gil-Díaz, Francisco, y Agustín Carstens (1996), “Algunas hipótesis relacionadas con la crisis mexicana de 1994-95”, Gaceta de Economía, ITAM, año 2 (3), otoño de 1996, pp. 5-67.

Granger, C. W. J., B. Huang, y C. W. Yang (2000), “A Bivariate Causality between Stock Prices and Exchange Rates: Evidence from the Recent Asia Flu”, The Quarterly Reviews of Economics and Finance, núm. 40, pp. 337-354.

Guzmán, María de la Paz, Soraya Leyva, y Antonio Cárdenas (2007), "La relación de causalidad entre el índice bursátil mexicano y el tipo de cambio spot", Análisis Económico, núm. 51, vol. xxII, tercer cuatrimestre.

Hernández, Ignacio, Arturo Morales, y Domingo Rodríguez (2007), "Eficiencia y volatilidad del mercado accionario en México", Comercio Exterior, noviembre, vol. 57, núm. 11, México.

Ibarrarán, V. Pablo, y Alfredo Troncoso (1998), "Causalidad entre el índice bursátil y el tipo de cambio en México", Gaceta de Economía, año 4, núm. 7, otoño, pp. 195-212.

Islas Camargo, Alejandro, y Francisco Venegas Martínez (2005), "Volatilidad de los mercados bursátiles de América Latina: efectos de largo plazo", Comercio Exterior, vol. 55, núm. 11, pp. 936-947.

Izquierdo Fernández, y Manzana Aragó (2002), “Transmisión de la volatilidad entre los mercados bursátiles desde la perspectiva de la bolsa española", Revista Electrónica de Economía Aplicada, Departamento de Finanzas y Contabilidad, versión preliminar, marzo, http//:www.revecap.com

Jensen, Michael C., y Kevin J. Murphy (1990), "Performance pay and top-management incentives", Journal of Political Economy, abril, vol. 98, núm. 2, pp. 225-264.

Johnson Christian, A., y Fabián Soriano A. (2004), "Volatilidad del mercado accionario y la crisis asiática", El Trimestre Económico, vol. 1 xxi (2), núm. 282, abril-junio, pp. 355-388.

Koutmos, G., C. Negakis, y P. Theodocciou (1993), "Sthocastic behavior of the Athens stock exchange", Applied Financial Economics, 3, pp. 119-26.

López Herrera, Francisco (2004), "Modelado de la volatilidad y pronóstico del índice de precios y cotizaciones de la Bolsa Mexicana", Contaduría y Administración, núm. 213, mayo-agosto, pp. 43-72.

Ludlow Wiechers, Jorge, y M. Beatriz Mota Aragón (2005a), "La dinámica de la volatilidad del IPC y sus componentes", REMEF, vol. 4, núm. 2, pp. 149-173. (2005 b), "Curvas de apalancamiento y elección de carteras en la BMV", REMEF, vol. 4, núm. 4, pp. 313-346.

Palacios, J. (1975), "The stock market in Spain; Tests of efficiency and capital market theory", in International Capital Markets, Elton y Gruber (eds.), North-Holland, Amsterdam, pp. 114-149. 
Qayyum, Abdul, y A. R. Kemal (2006), "Volatility Spillover Between the Stock Market and the Foreign Exchange Market in Pakistan", documento de discusión, PIDE.

Siourounis, Gregorios D. (2002), "Modelling volatility and testing for efficiency in emerging capital markets: the case of Athens stock exchange", Applied Financial Economics, 12, pp. 47-55.

Tsay, Ruey S. (2002), Analysis of Financial Time Series, John Wiley and Sons, Nueva York. 